\title{
Research on Mobile Information Service of Library
}

\author{
Zuobin Ning \\ The library of Southwest University for Nationalities, Chengdu City, Sichuan Province, 610041, China \\ ningzbinsc@gmail.com
}

\begin{abstract}
This paper mainly discusses the cloud computing in the library and should pay attention to the use of the advantages and problems in the application, and the paper puts forward the mode of constructing library information platform based on cloud computing, analyzes the basic architecture of cloud computing, in-depth study cloud computing in the library information platform, operation mode and cloud computing case, and prospects on cloud computing development and application in the future.
\end{abstract}

Keywords-cloud computing; Library; information platform; construction

\section{INTRODUCTIONS}

With development of information technology, it makes people more and more high requirements on the service quality of library, how to extend and improve the function of the library, to provide readers more convenient service is an objective of library human have been pursuing.

Due to the modern people's rhythm of life speeds up, increased traffic, carry heavy documentation and other reasons that makes the people to go to the library less and less; and browsing the university library website generally require the use of campus network and computer equipment, unable to use this for many. Campus network, it is not convenient to use a computer, so the utilization of library also has not high. Therefore, the traditional mode of library services has become increasingly difficult to meet the needs of the people.

High-speed development of mobile equipment for the library service provides a good platform for the user in recent years. Like mobile phone and other electronic mobile facility is almost the city people with tools, if we can use it without restrictions of time and space characteristics, to establish a "mobile library" on the foundation, can greatly expand the service scope of library, the convenience of readers. Because of this the mobile library system, the reader can realize the query personal information; download the electronic resources, library resources service whenever and wherever possible. Therefore, research on Mobile Library Based on WAP technology research has the practical significance, important based on this, and this paper researches and designs a mobile library technology based on WAP prototype system.

Cloud computing technology and mobile communication technology is a combination of the new trend. Mobile communication technology and the development of mobile Internet, a growing number of traditional Internet users start using mobile Internet services, and even some unused Internet users have begun to enjoy the mobile Internet services. In the past 2010, iphone 4 and ipad people through experience to bring triple play mobile connected life. Cloud computing simplifies IT infrastructure implementation, application cloud computing model will fundamentally change the library information acquisition, preservation methods and service concept, based on the traditional Internet, borrow books, browsing service and some personalized services, can be extended to the readers on the mobile terminal.

\section{The Hardware Structure Design Of The MobILe LIBRARY}

Wireless Application Protocol is a simplified wireless Internet protocol, in this paper, we propose the network topology structure of mobile WAP technology in Library Based on the graph, as shown in figure 1.

As you can see from Figure 1 the mobile library system architecture by the client (mobile phone), gateways and servers (library server group) consists of three parts ${ }^{[1-3]}$ : server (library server group, namely WAP content server) is a resource system existing library of various data types and data, can through the gateway (external WAP gateway) provide a variety of WAP page content of various information service; gateway (WAP gateway) plays the role of "translation" agreement, it will WAP client (mobile phone) and server (server) to connect, release and parsing WAP page content to the WAP users; and the client for various mobile devices that support the WAP protocol (such as mobile phones and other equipment).

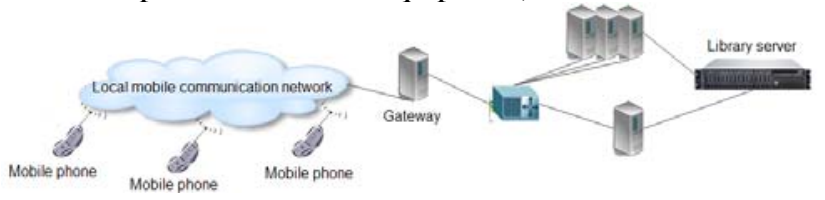

Figure 1 . The topology structure of mobile library system network

III. The StRUCtURE Of LiBRARy System BASED ON CLOUD-TECHNOLOGY

Mobile Library System Library System is just the application of a subsystem, which is in the public library system architecture to build up. Figure 2 is an overall model of cloud-based library system.

(A) Infrastructure layer

Infrastructure layer (Iaas), to provide high-level computing, data storage and network communications and other resources. That includes servers, storage and network devices. Provide computing, data storage and network communications and other virtual resources from the 
various virtual machines that make up the current mainstream virtual machine software with VMware, XEN, Virtual PC and Virtual-Box and so on.

(B) Platform services layer

The platform provides development environment built on the infrastructure layer, for developers, for the development of all types of cloud-based application software development environment for education and other public API, which provides PaaS. Web Service public API can be provided in the form to developers, such as Amazon Web Services, thus eliminating the developer of many system management operations. Developers can also use the public API to develop your own Web Service. These user-oriented services are available to the user either directly, or through a set of Open API.

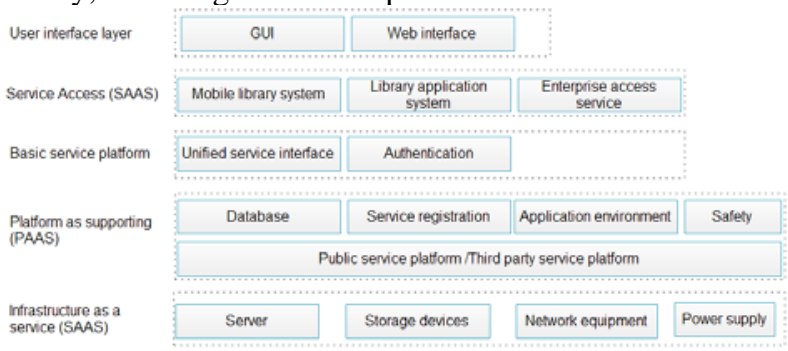

Figure 2. Library system model based on cloud computing

(C) Cloud service layer

Cloud services platform for the realization of crosscloud service integration, but also the realization of private and public cloud interoperability and form clouds of the key. It means Foreign Open API provides an abstraction of the application services. The platform library for different local systems, cloud computing services and third-party applications to integrate public services to a unified service interface methods available to the user, reducing the difficulty of integration services, enabling the formation of a highly integrated information platform library to unify the interface and operation mode for the user to provide services.

(D) The application service layer

Namely to provide library-oriented SaaS, the platform provides the ultimate direct terminal-oriented applications. We can build a data center infrastructure is cloud computing according to the principles of SOA. Therefore, it is a service-oriented infrastructure. It uses the principles of SOA architecture and put this principle applied to an infrastructure.

(E) User Interface Layer

For the user to access the client provides two interfaces: the traditional graphical user interfaces (GUI) and Web-based interface. With Web 2.0 technologies, services, mainly for B / S structure, so users only need to use a browser will be able to access the service. Traditional GUI Web interface as an auxiliary means of education for remote login on the cloud virtual machine access to the underlying resources

\section{The Process Of Cloud Services Platform IMPLEMENTATION}

Overall implementation process of digital Library cloud services platform shown in Figure 3.

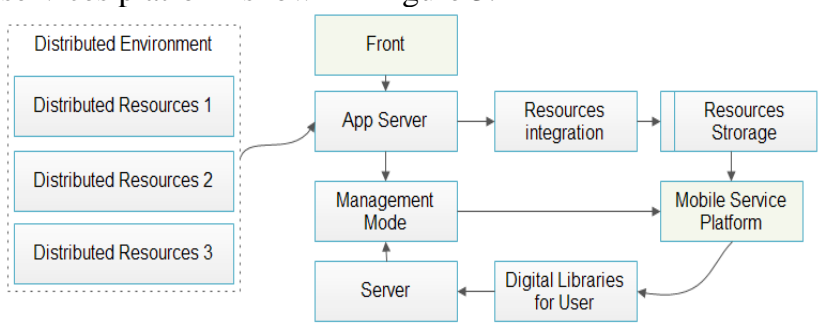

Figure 3. Flowchart of cloud services platform

Implementation process of cloud computing service platform was completed for the following four parts: (1) Front: for load balancing: static file requests forwarding and forwarding; (2) Application Server: The application server's main function is to run the program; (3) application Management node: management node role is to provide complex applications start and stop and billing; (4) server: provide various types of services - these services are integrated together.

\section{V.IMPLEMENTATION OF WAP TECHNOLOGY}

Design of WAP pages is a plain text file like WEB pages that can edit by using any text editor; the current operating environment is not good editor, the simple use of Windows notepad.

The system uses ASP to create a WML page. The WML page is created and HTML page similarity, difference lies in the file header format is not the same, a WML page header format [4]:

Response.ContentType $=$ "text/vnd.wap.wml; charset=GB2312"

Response.write ("Xmlversion=" 1 "?""

"? > <!" DOCTYPEwmlPUB-LIC "-//WAPFORUM//

"DTDWML1.1//EN"

"http://www.wapforum.org/DTD/wml 1

1.xml ">")

Response.write $("<$ wml $><$ card $>$ ")

The follow will introduce the system programming mode taking mobile search module as an example.

Because the WML page is the memory space constraints, the user through the display form in the process of browsing information, show 5 records at a time. If are not reading records after 5 records, prompting the user to continue reading, if the end, suggesting that read. Here StartP is used to specify a record number, DisP represents a number of records to show, the query results displayed as follows: 


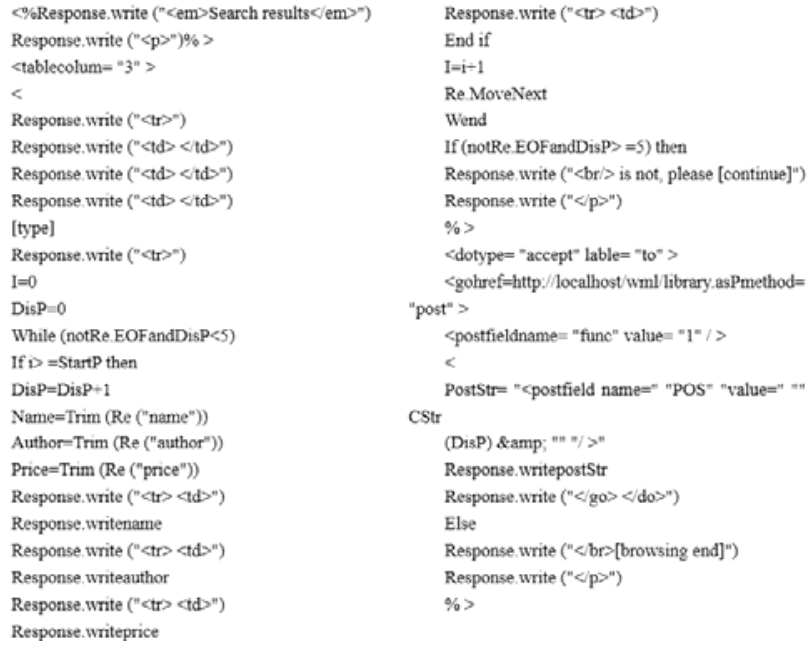

The above is the books retrieval module, then the other module construction is completed, a mobile library integrated system, then the test is carried out with Opera or Win-Wap browser, can be put into use after a successful test.

\section{CONCLUSIONS}

The rapid development of mobile devices has brought very good technology platform for mobile library, because it is not time and space constraints, equipment etc.. This paper successfully combines the WAP technology and the mobile equipment technology make the mobile library network into reality, so as to accelerate the shift to digital library service system the pace.

\section{ACKNOWLEDGEMENTS}

Funds: the basic scientific research business of Southwest University for Nationalities in 2014 central colleges "supported by the special fund project on the mobile information service of the libraries in Universities for Nationalities -- a case study of Southwest University for Nationalities" (project number: 2014SZYQN66)

\section{REFERENCES}

[1] Huang Hua. The research of information security countermeasures for Library Based on Cloud Computing [J]. The intelligence world books in primary and middle schools, 2010, (2): 58.

[2] Lin Ying, Sun Kuiming. The WAP library mobile information service system and application based on WAPOPAC[J]. Modern library and information technology, 2007 (9): 80-83

[3] Wang Yuheng, Qu Ying. Protocol of wireless application technology of WAP programming[J].Jiangsu electrical appliance, 2006 (3): 10-15

[4] Zhang Xinyuan, Meng Tao, Wang Long, et al. WML and its application in Digital Library[J].Journal of information, 2005 (2): $38-40$

[5] Lin Yishan. Application site in Library Service [J]. Library science research, 2007 (4): 37-39

[6] Xiao Peng. Dual impact of cloud computing on the library [J]. The study of Library Science,2009, (8): 43. 